\title{
NEW SOLUTIONS FOR HIGH-FREQUENCY HEARING IMPAIRMENT
}

\author{
Hugh J. McDermott \\ The Bionics Institute of Australia
}

Corresponding author: Hugh J. McDermott, The Bionics Institute of Australia, 384-388 Albert St., East Melbourne, VIC 3002, Australia, Phone: +61 39667 7526, Fax: +61 39667 7505, e-mail: hmcdermott@bionicsinstitute.org

\begin{abstract}
The common observation that hearing impairment tends to affect sound perception at high frequencies more than at lower ones has led recently to the development and evaluation of a number of innovative signal-processing techniques. These include frequency-lowering schemes that aim to provide improved audibility and discriminability of sound components by shifting them into a frequency range where the listener has less impairment. For people who have usable low-frequency hearing, but insufficient high-frequency hearing for effective use of a frequency-lowering hearing instrument, cochlear implantation is rapidly becoming a well-accepted option. The use of a cochlear implant, in combination with an acoustic hearing aid either in the implanted ear or the opposite ear, can provide large perceptual benefits, especially for understanding speech in noise and for listening to music. Selecting and fitting the most appropriate configuration of hearing devices is critical in maximising the perceptual benefits for both children and adults with severe high-frequency hearing impairment.
\end{abstract}

Key words: bimodal hearing $\bullet$ frequency compression • frequency transposition

\section{НОВЫЕ РЕШЕНИЯ ДЛЯ НАРУШЕНИЯ СЛУХА НА ВЫСОКИХ ЧАСТОТАХ}

\section{Резюме}

Было замечено, что нарушение слуха чаще происходит на высоких частотах, чем на низких, поэтому в последнее время все больше усилий прилагается к развитию и оценке многих инновационных, обрабатывающих сигналы методов. Они включают в себя схемы,

понижающие частоту, которые могут обеспечить улучшенную слышимость и способность определения звуковых компонентов, благодаря их перемещению на те частоты, на которых пациент лучше слышит. В случаях, когда у людей слух на низких частотах является достаточным, а на высоких не удовлетворительным для эффективного использования решения, понижающего слух на низкие частоты, все чаще используется кохлеарная имплантация.

Использование кохлеарного импланта вместе с акустическим слуховым аппаратом на имплантированном или другом ухе, может обеспечить значительные перцепционные возможности, особенно для понимания речи в шуме и для того, чтобы слушать музыку. Выбор и настройка соответствующей конфигурации устройств важны для увеличения перцепционных возможностей детей и взрослых с серьезными нарушениями слуха на высоких частотах.

Ключевые слова: бимодальный слух • сжатие частоты • перемещение частоты

\section{NUEVAS SOLUCIONES EN CASO DE SORDERA EN LAS ALTAS FRECUENCIAS}

La observación común, que la sordera afecta la percepción de los sonidos en las altas frecuencias más frequentamente que en los bajos, ha resultado recientemente en el desarrollo y la evaluación de varias técnicas innovadoras que tratan la señal. Ellos incluyen

los esquemas que bajan las frecuencias para mejorar la audibilidad y discriminabilidad de sonidos cambiandolos en un rango de frecuencia donde el oyente tiene el daño menos grave. En caso de la gente que tiene el oído utilizable en las frecuencias bajas pero insuficiente en las altas para el uso efectivo de un instrumento que baja las frecuencias del oído, implantación coclear es una opción más frequentemente usada. El uso de una implantación coclear en combinación con un audífono acústico en la oreja implantada o no, puede dar ventajas grandes, sobre todo para entender el discurso en el ruido y para escuchar música. La selección y la programación de la configuración más apropiada de los dispositivos auditivos son críticas en maximizar la beneficia perceptual tanto en caso de niños como de adultos con sordera grave en las frecuencias altas.

Palabras claves: audiencia de bimodal • compresión de frecuencia • transposición de frecuencia 


\section{Background}

Every experienced audiologist knows that one of the most common types of hearing loss is sensorineural impairment that affects the high frequencies more than the low frequencies. In many instances, the extent and severity of high-frequency hearing impairment is such that simple amplification of sounds by a conventional hearing aid (HA) is insufficient to restore normal hearing acuity. In some cases, amplification may fail to make certain high-frequency sounds audible, let alone identifiable. Excessive amplification, leading to abnormally high levels in the impaired ear, can sometimes result in distortion and poor sound quality. There is even evidence that people with particular types of hearing impairment may understand less speech when a HA is fitted to provide high-frequency amplification in comparison with a fitting restricted to a narrower bandwidth that avoids such amplification. This effect can occur when there are 'dead regions' in the cochlea which are unable to convert sound vibrations into neural activity. When extensive dead regions are located in the basal part of the cochlea, perception of high-frequency components of sounds may be deleteriously affected [1].

Recent technological developments are enabling better outcomes to be achieved for many people with this kind of hearing loss. In particular, several types of digital HA now provide sophisticated techniques for lowering frequencies in real time. Such techniques may help listeners with highfrequency impairment to detect and discriminate components of sounds having high frequencies. For example, certain unvoiced fricative consonants in speech, such as /s/, are characterised acoustically by an energy distribution that may be concentrated at frequencies as high as $5-15 \mathrm{kHz}$ but have little energy below $4 \mathrm{kHz}$. It is important for people to be able to discriminate and identify those consonants. In English, for instance, /s/ is used to denote the plural number (e.g., "bat" versus "bats"), the possessive case ("bat's"), some contractions ("it is" = "it's"), and the third-person singular present tense of verbs ("she bats"). Not only speech sounds but various environmental noises are dominated by high-frequency components. On the other hand, many common types of background noise have most acoustic energy at low frequencies. The masking of speech phonemes by low-frequency noise, such as that heard inside a car, is a further reason for attempting to improve the audibility and salience of high-frequency sound signals in general.

For people whose hearing impairment is so severe that even frequency lowering in an acoustic HA cannot provide sufficient help, there are now new opportunities for applying electrical stimulation. Although the cochlear implant (CI) is already a widely accepted treatment for people with severe to total hearing loss in both ears, recent research is showing that CIs can also be beneficial for people with usable low-frequency hearing. This article discusses briefly the use of acoustic hearing in combination with a CI as well as the application of frequency-lowering schemes in hearing aids.

\section{Frequency Lowering}

The idea that lowering high frequencies into a region of hearing where they can be made more audible and more easily discriminated is not new. However, the advantages of the latest digital signal-processing technology in making complex frequency-lowering schemes practical have led to new implementations based on this principle. Recently two innovative schemes have been released commercially. The first, from Widex, is a linear frequency transposition (LFT) scheme known as the 'Audibility Extender'. The other is a non-linear frequency compression (NLFC) scheme called 'SoundRecover' that is provided by Phonak. Although they are both potential solutions to the same underlying problem (i.e., poor perception of high-frequency sounds by some people with hearing impairment), they function in very different ways.

The Widex LFT processing operates on two defined frequency regions designated the source octave and the target octave. Frequency components in the source octave are analysed and a dominant peak is selected. This peak is shifted down in frequency, typically by one octave. Surrounding frequency components in the source octave are also shifted down at the same time, but the size of the shift applied to them is defined as a constant number of hertz. For example, if the peak in the source octave is at $4 \mathrm{kHz}$, it will be lowered by one octave to $2 \mathrm{kHz}$. As the amount of this shift is $2 \mathrm{kHz}$, all frequencies in the source octave will simultaneously be lowered by $2 \mathrm{kHz}$. This means, for instance, that a source frequency of $3.5 \mathrm{kHz}$ would be lowered to $1.5 \mathrm{kHz}$, which is not exactly a one-octave shift. Because this linear frequency-shifting process, when applied to each frequency in the source octave, may result in a target bandwidth that is wider than one octave, the output of the process is filtered to limit it to one octave. Lowered signals in the target octave are mixed with any signals already present in that frequency region. Amplification and other appropriate processes are then applied as usual. Once enabled during fitting, the LFT processing is active all the time, although the frequencies of the source and target octaves can be selected to suit individual HA users.

In contrast, the Phonak NLFC processing does not depend on any features of the incoming sound (such as the dominant peak frequency in the LFT source octave). Instead, all frequencies above a preset cut-off frequency are lowered by progressively increasing amounts. The steepness across frequency of this progressive lowering is determined by a frequency-compression ratio. For example, if the cut-off is set to $2 \mathrm{kHz}$, and the ratio is $2: 1$, each octave range of input frequencies above $2 \mathrm{kHz}$ will be compressed into a half-octave range. Thus the input range $2-4 \mathrm{kHz}$, which is one octave wide, will become $2-2.8 \mathrm{kHz}$ (half an octave) at the output of the HA. All frequencies below the cut-off are unchanged by the processing, and there is no overlap or mixing of shifted frequencies with any lower frequencies that may be present at the same time. When NLFC is enabled during fitting, the cut-off frequency and compression ratio are preselected automatically based on the audiogram of the HA user. Generally, lower cut-off frequencies and larger compression ratios are recommended for audiograms that show worse hearing at mid to high frequencies, or a steeper downward slope. However, a relatively high cut-off and ratio just above 1:1 might be suitable for people with audiograms that are almost flat or even slightly upward-sloping. In any case, the parameters of NLFC 


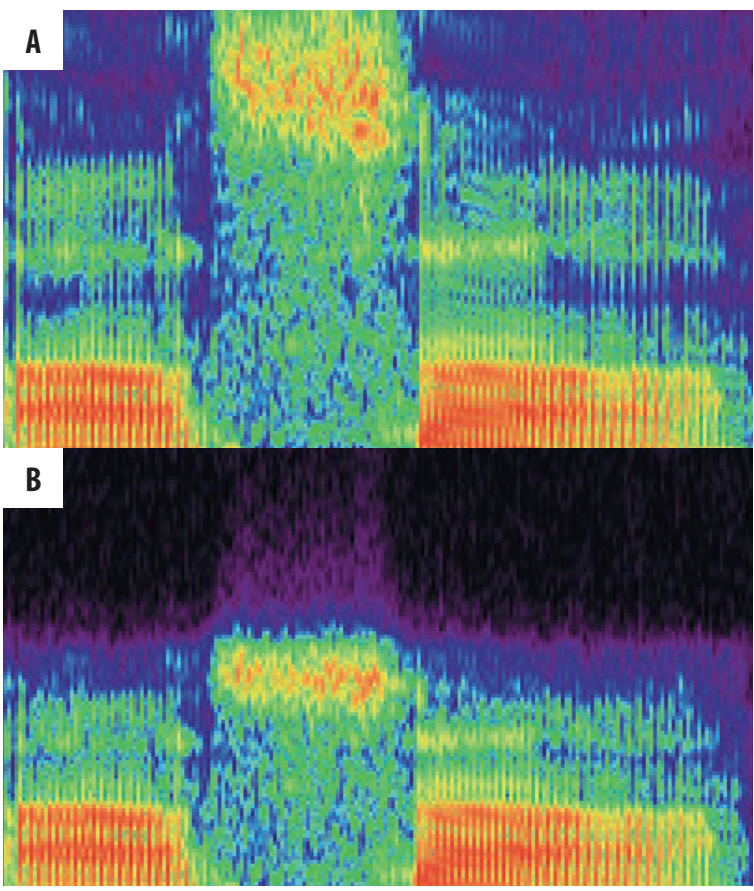

Figure 1. Spectrograms of speech sounds before and after processing with the Phonak 'SoundRecover' NLFC scheme. (A) the utterance /asa/, showing mostly low-frequency energy for the two vowel sounds and higher-frequency energy for the fricative consonant. (B) the same utterance after processing by NLFC, showing that the vowel sounds are almost unchanged, whereas the energy associated with the consonant has been shifted down and compressed into a narrower frequency range. The NLFC settings were: cutoff frequency $=2 \mathrm{kHz}$; compression ratio $=2: 1$. In each panel, the vertical axis shows frequency with a range of $0-8 \mathrm{kHz}$, while the horizontal axis shows time with a range of approximately $0.7 \mathrm{~s}$. Warmer colors indicate parts of the acoustic signal with relatively more energy. Images provided by Phonak AG.

can easily be adjusted during HA fitting to suit the individual listener. As with LFT, NLFC operates all the time after initial activation.

Examples of speech sounds processed by each of the above schemes are illustrated in Figures 1 and 2. In the left panel of Figure 1, a spectrogram of the utterance /asa/ is shown. It can be seen that the two vowel sounds have most energy at frequencies below about $2 \mathrm{kHz}$, whereas the dominant energy in the fricative consonant covers a range of about $5-8 \mathrm{kHz}$. In the right panel, the same utterance is shown after processing by the Phonak NLFC scheme with a cutoff of $2 \mathrm{kHz}$ and a frequency compression ratio of 2:1. This setting of the NLFC parameters means that the vowel sounds are largely unaffected by the processing. In contrast, the consonant sound has been shifted down and compressed in frequency to a range of approximately $3-4 \mathrm{kHz}$.

Figure 2 shows a spectrogram of the word "fish" after processing by the Widex LFT scheme, with the start frequency of the source octave set to $2.5 \mathrm{kHz}$ and the target octave set below the source by one octave. The energy in

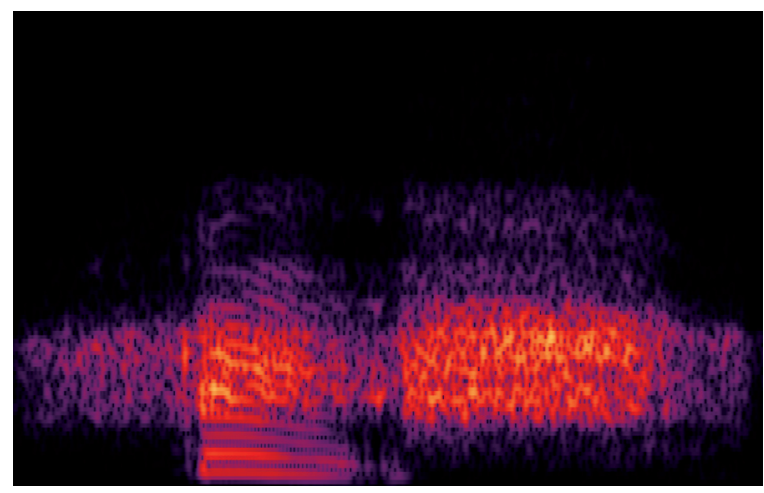

Figure 2. Spectrogram of the word "fish" after processing with the Widex 'Audibility Extender' LFT scheme, plotted in a format similar to that of Figure 1. The figure shows that the fricative consonants /f/ and / / have been shifted down into a frequency range that partially overlaps that of the vowel. For the particular settings of the LFT program used to generate this spectrogram, most energy in the consonants after processing is in the frequency range $1.25-2.5 \mathrm{kHz}$.

the consonants /f/ and / $/$, which was originally distributed across frequencies above approximately $2 \mathrm{kHz}$, has been shifted into the range $1.25-2.5 \mathrm{kHz}$. Although some of the higher-frequency components of the vowel sound, particularly the second formant around $2.5-3 \mathrm{kHz}$, have also been lowered by the LFT processing, the lower formant region has not been affected. As would be expected with this setting of the LFT parameters, there is little output energy above about $3 \mathrm{kHz}$ for any phoneme.

Unfortunately, there do not appear to be any publications so far that report on a direct perceptual comparison between these two frequency-lowering schemes. However, recent publications suggest that the Phonak SoundRecover NLFC scheme can be beneficial for many adults and children with sloping, high-frequency hearing impairment [2], and that the Widex Audibility Extender LFT scheme may help some adults with severe to profound hearing loss recognise consonants after training [3]. Considering the main acoustic effects of each type of processing as illustrated in Figures 1 and 2, it may be that an initial selection of appropriate candidates for each scheme could be based on the configuration of the audiogram. For example, if an individual with hearing impairment has no useful hearing sensitivity above a relatively low frequency, such as $2 \mathrm{kHz}$, it may be that the LFT scheme will present more information from higher frequencies into that very restricted hearing bandwidth. On the other hand, if such an individual does have some usable acoustic sensitivity at higher frequencies, then it is plausible that an appropriate setting of the NLFC scheme would improve the perception of high-frequency sounds without also creating audible artifacts or distortion. Until independent and reliable comparative studies on these processing schemes are published, it may be that audiologists should regard each scheme as having potential to help at least some HA users, and particularly those with impairments affecting mainly the high frequencies. However, if frequency-lowering schemes do not help, or individuals have hearing impairment that is severe or worse across a wide frequency 
range in both ears, then a cochlear implant is likely to be a more effective solution. In such cases, extensive dead regions are probably present in each cochlea, whereas there is no evidence so far to suggest that the existence of dead regions is a prerequisite for a successful fitting of a frequency-lowering hearing aid.

\section{Cochlear Implants}

Today's cochlear implants provide up to 22 intracochlear electrodes that stimulate residual auditory neurons electrically. They bypass the normal mechanical filtering and transduction processes in the cochlea, and therefore can create hearing sensations even in ears that are so deaf that the hair-cell population is minimal or absent. Although such ears cannot be aided satisfactorily with any type of acoustic HA, modern multichannel CI devices enable the majority of recipients to understand nearly all speech, provided that there is no background noise [4]. However, their performance in more-complex acoustic situations, such as listening to music or speech in competing noise, is often unsatisfactory [5]. To understand this problem, it might help to imagine an analogy.

The deaf cochlea can be thought of as a blank canvas, while the function of a CI is to paint a picture on that canvas. The picture created is equivalent to hearing a recognisable sound. For a widely used CI system with 22 electrodes, each electrode can be represented by a spray-can of paint. Because the electrodes are not actually in contact with the auditory nerves in the cochlea, the spray-cans can be imagined as located some distance from the canvas. What kind of picture can be painted in this way?

It should be straightforward to create a cartoon-like sketch, which conveys symbolic meaning but not much else. This is analogous to understanding speech, in which the intended meaning is more important to perceive than the sound quality. Unfortunately, however, it would not be possible to paint an intricate landscape or detailed portrait, for example. This is not only because there are too few sources of paint, but also because the paint spreads out from the spray-cans, resulting in a blurry image. A further problem is that the canvas is often imperfect; it may be torn or incomplete. This is analogous to the patchy loss of auditory neurons that often results from long-term deafness and the occurrence of cochlear dead regions.

Future cochlear implants need to have many more electrodes if their users' perception of complex sounds, including music, is to be greatly enhanced. Furthermore, those electrodes must be placed closer to the auditory nerves so that the stimulation they deliver can be focused more precisely. At present, it is not clear how many electrodes would be required if the eventual aim is to approximate normal hearing. In a normal cochlea, the process of converting sound vibrations into neural activity is performed by many thousands of hair-cells which are distributed along the length of the cochlear spiral. Taking this fact as a guide, it seems likely that the number of electrodes may need to be increased by a factor of at least ten - perhaps even a hundred - relative to today's implants for large performance improvements to be achieved. Such devices are not likely to be available for routine implantation for many years.

\section{Bimodal Hearing}

In the meantime, research is showing that the use of acoustic hearing in combination with cochlear implants is highly beneficial. Such a combination is commonly referred to as bimodal hearing, and, in the following, this term will be used to describe any device configuration that enables simultaneous use of acoustic and electric hearing. The increasing usage of bimodal hearing is significant, particularly because the high level of performance possible with the best existing CI devices is leading to a rapid expansion in the number of CI recipients who are not totally deaf. When partially deaf implant users listen simultaneously via the $\mathrm{CI}$ and one or two conventional hearing aids, they report that their understanding of speech in noisy conditions and their appreciation of music are both enhanced $[6,7]$. Researchers are now developing improved soundprocessing systems and fitting techniques to maximise the benefit available from such combined hearing devices. For example, one existing scheme, known as Adaptive Dynamic Range Optimisation (ADRO), has been shown to benefit listeners who use a CI and a HA together [8]. ADRO has been available for some years in both CI sound processors and certain types of HA, although originally it was developed specifically for bimodal applications. It functions automatically to adjust the levels of sounds independently in multiple frequency bands so as to maximise audibility while maintaining listening comfort. As it is widely appreciated that balancing loudness between devices as far as possible is helpful to listeners with hearing impairment, it is not surprising that $\mathrm{ADRO}$, which makes such adjustments in real time, can be beneficial to many bimodal device users.

The recent and continuing increase in the number of bimodal CI users has encouraged development of electrode arrays and surgical techniques that have a greater likelihood of preserving residual hearing. In the past, it has been assumed that implantation of a multi-electrode array into a cochlea would inevitably destroy any residual acoustic hearing sensitivity. Although in fact there are numerous recipients of conventional CIs who have retained some hearing in the implanted ear, it is far more common for bimodal device users to have the acoustic HA in the ear opposite that containing the CI. However, the most usual configuration of hearing impairment is characterised by having better acoustic sensitivity at low frequencies than at high frequencies. This is fortuitous in that CI electrode arrays are inserted surgically from an opening near the base of the cochlea, and typically do not extend all the way to the cochlear apex. Therefore, if there exist hair-cells that provide some acoustic sensitivity to low-frequency sounds, they will be located near the apex in a cochlear region that may not be affected by the electrode array. To exploit further this opportunity, researchers and CI manufacturers have developed arrays that are shorter than the conventional ones, and can be inserted into the cochlea with less surgical trauma. In cases where the use of these devices has resulted in good preservation of acoustic hearing, it is possible for recipients to perceive sounds acoustically in both ears rather than only in the non-implanted ear. A device or arrangement of devices that provides both acoustic and electric stimulation in the same ear is sometimes referred to as a hybrid system. Recent publications have 


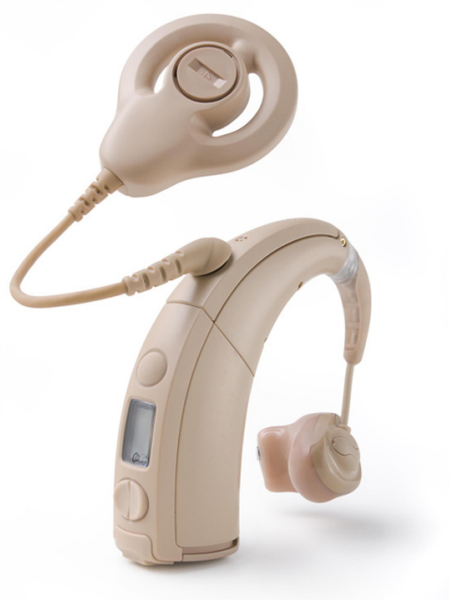

Figure 3. A hybrid sound processor from Cochlear Ltd. Intended for use by cochlear implant recipients with usable acoustic hearing in the implanted ear, this processor can control a $\mathrm{Cl}$ to generate electric stimulation via the coil seen at the top of the picture, while acoustic signals are available simultaneously from the transducer seen at the lower right. Image provided by Cochlear Ltd.

reported that CI devices with short electrodes have enabled better preservation of acoustic hearing than conventional devices. Because the perceptual outcomes of these systems are generally better than would have been expected with use of a conventional CI device in combination with a HA in only the opposite ear, the range of audiogram configurations now thought suitable for possible implantation has expanded. People with normal or nearnormal hearing thresholds in both ears up to at least 500 $\mathrm{Hz}$ have become successful users of short-electrode CI systems [9-11], although it is more common for CI candidates to have a hearing loss of moderate or worse severity at these frequencies. Figure 3 shows a recent Hybrid CI sound processor that has been developed by Cochlear Ltd.

The ongoing research outlined above is creating an everexpanding range of new options for people with high-frequency hearing loss. Audiologists who diagnose hearing impairment and recommend suitable treatments can now select from several different potential solutions. In most instances, effective assistance will be provided by fitting a modern acoustic hearing aid. In addition, the use of a frequency-lowering sound-processing scheme will improve the perception of many HA users. Finally, in cases where it is not feasible for an acoustic hearing instrument to provide adequate audibility particularly for high-frequency sounds, a cochlear implant may be a more appropriate solution. Whenever there is usable acoustic hearing in an implant recipient, the use of one HA or two HAs in combination with the $\mathrm{CI}$ is likely to be beneficial.

\section{Conclusions}

- The common occurrence of high-frequency hearing impairment has led to the introduction of innovative sound-processing schemes in acoustic HAs and to the development of new CI devices.

- Nonlinear frequency compression (NLFC) and linear frequency transposition (LFT) function in different ways to improve the perception of high-frequency sounds by adults and children with suitable types of hearing impairment [12].

- Although there is some evidence that both NLFC and LFT may provide perceptual benefits to suitable HA users, it is not yet clear whether either scheme may be more appropriate or more effective for particular listeners, and if so, how the better scheme should be selected and fitted for each individual.

- As it is becoming progressively more common for people who have hearing that cannot be aided satisfactorily by an acoustic HA to receive a CI, new bimodal hearing systems are being developed and evaluated.

- Bimodal hearing can help many CI users to appreciate music and understand speech in noise better than is typical for recipients of conventional CIs who have no usable acoustic hearing.

- The development of short electrode arrays and lesstraumatic surgical implantation procedures is increasing the probability that future bimodal device users will have acoustic hearing in both ears rather than only in the ear opposite the CI.

- The good perceptual performance of the latest CI systems and the reduced risk of hearing loss in the implanted ear resulting from the surgery are encouraging further expansion of the audiological criteria for CI candidacy.

- It may soon be routine for people with up to normal hearing thresholds at low frequencies in both ears, but no usable hearing at relatively high frequencies in at least one ear, to receive a hybrid CI so as to benefit from bimodal hearing binaurally.

\section{Acknowledgements}

A version of this article was first published in Canadian Hearing Report, and the assistance of that journal's publishers with the present publication is gratefully acknowledged. The helpful comments of two anonymous reviewers on a previous version of the manuscript are appreciated. The Bionics Institute acknowledges the support it receives from the Victorian Government through its Operational Infrastructure Support Program.

\section{References:}

1. Moore BCJ: Dead regions in the cochlea: Diagnosis, perceptual consequences, and implications for the fitting of hearing aids. Trends Amplif, 2001; 5(1): 1-34
2. Glista D, Scollie S, Bagatto M et al: Evaluation of nonlinear frequency compression: Clinical outcomes. Int J Audiol, 2009; 48(9): 632-44 
3. Kuk F, Keenan D, Korhonen P, Lau C-c: Efficacy of linear frequency transposition on consonant identification in quiet and in noise. J Am Acad Audiol, 2009; 20(8): 465-79

4. Helms J, Weichbold V, Baumann U et al: Analysis of ceiling effects occurring with speech recognition tests in adult cochlear-implanted patients. ORL J Otorhinolaryngol Relat Spec, 2004; 66(3): 130-35

5. McDermott HJ: Music perception with cochlear implants: A review. Trends Amplif, 2004; 8(2): 49-82

6. Ching TY, van Wanrooy E, Dillon H: Binaural-bimodal fitting or bilateral implantation for managing severe to profound deafness: A review. Trends Amplif, 2007; 11(3): 161-92

7. McDermott HJ: Cochlear implants and music. In: Chasin M (ed.), Hearing Loss in Musicians, Prevention and Management. San Diego, CA: Plural Publishing, 2009; 117-27
8. Blamey P, Saunders E: A review of bimodal binaural hearing systems and fitting. Acoustics Australia, 2008; 36(3): 87-91

9. Gantz BJ, Hansen MR, Turner CW et al: Hybrid 10 clinical trial. Audiol Neurotol, 2009;14(Suppl.1): 32-38

10. Skarzynski H, Lorens A, Piotrowska A, Podskarbi-Fayette R: Results of partial deafness cochlear implantation using various electrode designs. Audiol Neurotol, 2009; 14(Suppl.1): 39-45

11. Prentiss S, Sykes K, Staecker H: Partial deafness cochlear implantation at the University of Kansas: Techniques and outcomes. J Am Acad Audiol, 2010; 21: 197-203

12. McDermott HJ: A technical comparison of digital frequencylowering algorithms available in two current hearing aids. PLoS ONE 2011; 6(7): e22358. DOI: 10.1371/journal.pone.0022358 